ESAIM: PROCEEDINGS AND SURVEYS, September 2014, Vol. 45, p. 138-147

J.-S. Dhersin, Editor

\title{
2D NUMERICAL SIMULATION \\ OF A LOW MACH NUCLEAR CORE MODEL WITH STIFFENED GAS USING FREEFEM++
}

\author{
Stéphane Dellacherie ${ }^{1}$, Gloria FaCCanoni $^{2}$, Bérénice Grec ${ }^{3}$, Ethem Nayir $^{3}$ \\ AND YOHAN PENEL ${ }^{4}$
}

\begin{abstract}
We investigate a simplified model describing the evolution of the coolant within a nuclear reactor core (e.g. of PWR type). This model is named LMNC (for Low Mach Nuclear Core) and consists of the coupling between three equations of different types together with boundary conditions specific to the nuclear framework. After several articles dedicated to dimension 1, we present in this paper some monophasic two-dimensional numerical results when the fluid is modelled by the stiffened gas law describing the pure liquid phase. The underlying numerical strategy is based on the Finite-Element software FreEFEM ++ .
\end{abstract}

Résumé. Nous étudions dans ce document un système d'équations modélisant le comportement simplifié du fluide caloporteur dans un cœur de réacteur nucléaire (par exemple de type REP). Ce modèle a pour nom LMNC (pour Low Mach Nuclear Core) et se compose de trois équations de natures différentes couplées avec des conditions aux limites spécifiques au domaine nucléaire. Après plusieurs articles dédiés à l'analyse du modèle en dimension 1, nous présentons ici des résultats en dimension 2 pour des écoulements monophasiques modélisés à l'aide de la loi des gaz raidis pour la phase liquide. Ces résultats sont obtenus grâce au code FreEFEM ++ basé sur la méthode des Éléments Finis.

\section{INTRODUCTION}

The modelling of nuclear reactors is hard to achieve since it requires the coupling of several multi-scale multiphysics problems [6]. Indeed, a reactor is characterized by a large number of systems corresponding to different functions (heating, cooling, energy production, ...). The natural process is thus to split the problems into lower-scale ones and then to carry out the coupling between them [11]. Moreover, there exist several industrial

\footnotetext{
${ }^{1}$ DEN/DANS/DM2S/STMF, Commissariat à l'Énergie Atomique et aux Énergies Alternatives - Saclay, 91191 Gif-sur-Yvette, France \& e-mail: stephane.dellacherie@cea.fr

2 Université de Toulon - IMATH, EA 2134, avenue de l'Université, 83957 La Garde, France

\& e-mail: faccanoni@univ-tln.fr

3 MAP5 UMR CNRS 8145 - Université Paris Descartes - Sorbonne Paris Cité, 45 rue des Saints Pères, 75270 Paris Cedex 6, France \& e-mail: berenice.grec@parisdescartes.fr \& e-mail: ethemnayir@gmail.com

${ }^{4}$ CEREMA-INRIA - team ANGE and LJLL UMR CNRS 7598, 4 place Jussieu, 75005 Paris, France

\& e-mail: yohan.penel@cerema.fr
}

(C) EDP Sciences, SMAI 2014 
codes based on a compressible model providing numerical approximations of the whole nuclear reactor (see for instance $[1,4])$.

A model was proposed in [7] to describe specifically flows within the reactor core in a simplified approach (which may be enriched to provide a more complete description of the overall process). Based on the assumption that the average Mach number is small, the model was derived through an asymptotic expansion performed in the monophasic compressible Navier-Stokes equations with an energy source term. The readers may refer to $[12,15]$ for similar processes leading to low Mach number models. This asymptotic approach amounts to filtering out the acoustic waves. Consequently, the mathematical nature of the resulting system of PDEs is modified which requires numerical methods that are different from the compressible Navier-Stokes framework. The model derived in [7] is called LMNC (for Low Mach Nuclear Core model) and consists of a transport equation upon a thermodynamic variable (here the total enthalpy), of an elliptic divergence constraint upon the velocity (with a source term which underlines the compressible property of the flow) and of the parabolic momentum equation. It thus exhibits a structure similar to the incompressible Navier-Stokes equations for which several Finite Element algorithms have been designed in the literature [16].

Applications to low Mach number configurations have been achieved for instance in [9]. Our study differs from the latter reference due to the model - which, in our case, is modified to match the low Mach regime (like in [5]) - and due to the boundary conditions imposed by the underlying nuclear framework. The structure of the equations lead to explicit analytic solutions [3] when the phases are modelled by the stiffened gas law even when phase transition is involved. Notice that the present paper is restricted to monophasic flows as we aim at assessing a new numerical approach. 1D simulations were performed in $[2,3,8]$ by means of a numerical scheme based on the method of characteristics. The latter algorithm strongly relies on the decoupling of the equations which is only valid in dimension 1 . It could though be extended to dimension 2 through an explicit treatment. However, we did not select this strategy in the present work. We rather choose to apply the Finite Element method directly by using the free software FreEFEM $++[10]$. This robust tool enables to deal with the aformentioned boundary conditions. Contrary to [9], the convective part of the equations is not treated directly through the weak formulation but by means of the method of characteristics [14]. We emphasize that no theoretical study of the Finite Element approach is carried out in this paper as we only focus on numerical simulations.

The paper is organized as follows. The model is presented in Section 2 including the initial and boundary conditions. Its weak formulation is given in Section 3 as well as the resulting algorithm. Academic numerical examples are then provided in Section 4, namely an intrinsically 1D flow (which enables to legitimate the numerical strategy) and a pure 2D flow.

\section{Governing EQUATions}

The model is set in the rectangular domain $\Omega=\left\{\boldsymbol{x}=(x, y) \in\left[0, L_{x}\right] \times\left[0, L_{y}\right]\right\}$. The $2 \mathrm{D}$ nonconservative formulation of the LMNC model [7] reads

$$
\left\{\begin{array}{l}
\nabla \cdot \mathbf{u}=\frac{\beta\left(h, p_{0}\right)}{p_{0}} \Phi(t, \boldsymbol{x}), \\
\rho\left(h, p_{0}\right) \cdot\left(\partial_{t} h+\mathbf{u} \cdot \nabla h\right)=\Phi(t, \boldsymbol{x}), \\
\rho\left(h, p_{0}\right) \cdot\left(\partial_{t} \mathbf{u}+(\mathbf{u} \cdot \nabla) \mathbf{u}\right)-\nabla \cdot \sigma(\mathbf{u})+\nabla \bar{p}=\rho\left(h, p_{0}\right) \mathbf{g},
\end{array}\right.
$$

where $\mathbf{u}=(u, v)$ and $h$ denote respectively the velocity field and the total enthalpy of the fluid. This model is characterized by two pressure fields. This decomposition results from the filtering out of the acoustic waves. The thermodynamic pressure $p_{0}$ is involved in the equation of state and is an average pressure (constant in time 
and space) within the core. The dynamic pressure $\bar{p}$ appears in the momentum equation and can be considered a perturbation around $p_{0}$. We mention that model (1) is only valid under the assumption that pressure $p_{0}$ does not depend on time.

The stress tensor $\sigma(\mathbf{u})$ models viscous effects: the classic internal friction in the fluid as well as the friction on the fluid due to technological devices in the nuclear core (e.g. the friction on the fluid due to the fuel rods). In the sequel, we take

$$
\sigma(\mathbf{u})=\mu\left(h, p_{0}\right)\left(\nabla \mathbf{u}+(\nabla \mathbf{u})^{T}\right)+\eta\left(h, p_{0}\right)(\nabla \cdot \mathbf{u}) \mathbb{I},
$$

where $\mu$ and $\eta$ are the viscosity coefficients determined by constitutive laws. Other choices are possible depending on the modelling scale at stake.

The power density $\Phi(t, \boldsymbol{x})$ is a given function of time and space modelling the heating of the coolant fluid due to the fission reactions in the nuclear core. Finally, $\mathrm{g}$ is the gravity field.

To close the system, we have to specify the equation of state (EOS) that relates the density $\rho$ to the unknowns of System (1). In the present work, the properties of the monophasic fluid are prescribed by the stiffened gas law:

$$
\rho\left(h, p_{0}\right)=\frac{\gamma_{\ell}}{\gamma_{\ell}-1} \frac{p_{0}+\pi_{\ell}}{h-q_{\ell}}
$$

where $\gamma_{l}, q_{\ell}$ and $\pi_{\ell}$ are characteristic constants of the liquid phase [3, Table 1].

This relation yields the expression of the compressibility coefficient involved in Equation (1a)

$$
\beta\left(h, p_{0}\right) \stackrel{\text { def }}{=}-\frac{p_{0}}{\rho^{2}\left(h, p_{0}\right)} \cdot \frac{\partial \rho}{\partial h}\left(h, p_{0}\right)=\beta_{\ell}\left(p_{0}\right) \stackrel{\text { def }}{=} \frac{\gamma_{\ell}-1}{\gamma_{\ell}} \frac{p_{0}}{p_{0}+\pi_{\ell}} .
$$

The coefficient is constant in the case of this equation of state.

\section{Boundary and initial conditions}

The fluid is injected at the bottom of the core $(y=0)$ at a given enthalpy $h_{e}$ and at a given flow rate $D_{e}$. Without viscous effects, it is possible to impose the dynamic pressure $\bar{p}$ at the exit of the core $\left(y=L_{y}\right)$ as in [3]. When viscosity is taken into account, the nature of the equations is different and more information is needed at the exit. We chose here a free outflow boundary condition.

More precisely, the boundary conditions (BC) at the bottom of the domain are

$$
\left\{\begin{array}{l}
h(t, x, 0)=h_{e}(t, x) \\
(\rho \mathbf{u})(t, x, 0)=\left(0, D_{e}(t, x)\right)
\end{array}\right.
$$

and at the top of the domain we consider free outflow conditions

$$
(\sigma(\mathbf{u}) \mathbf{n}-\bar{p} \mathbf{n})\left(t, x, L_{y}\right)=\mathbf{0},
$$

where $\mathbf{n}$ is the unit normal vector. On the lateral walls we consider free-slip condition, i.e.

$$
\left\{\begin{array}{l}
(\mathbf{u} \cdot \mathbf{n})(t, 0, y)=(\mathbf{u} \cdot \mathbf{n})\left(t, L_{x}, y\right)=0 \\
(\sigma(\mathbf{u}) \mathbf{n} \cdot \boldsymbol{\tau})(t, 0, y)=(\sigma(\mathbf{u}) \mathbf{n} \cdot \boldsymbol{\tau})\left(t, L_{x}, y\right)=0
\end{array}\right.
$$

where $\boldsymbol{\tau}$ is some unit tangential vector. 
As for the initial state, it is prescribed by

$$
\left\{\begin{array}{l}
h(0, \boldsymbol{x})=h_{0}(\boldsymbol{x}), \\
\mathbf{u}(0, \boldsymbol{x})=\mathbf{u}_{0}(\boldsymbol{x}), \\
\bar{p}(0, \boldsymbol{x})=0 .
\end{array}\right.
$$

\section{Hypotheses}

Several assumptions are made to ensure that the problem is well-posed and has a physical meaning. As for the data, we suppose that:

(i) $\Phi(t, \boldsymbol{x})$ is nonnegative for all $(t, \boldsymbol{x}) \in \mathbb{R}^{+} \times\left[0, L_{x}\right] \times\left[0, L_{y}\right]$;

(ii) $p_{0}$ is a positive constant.

Hyp. (i) characterizes the fact that we study a nuclear core where the coolant fluid is heated.

The second hypothesis concerns the modelling parameters:

(iii) $\gamma_{\ell}>1$;

(iv) $\pi_{\ell}$ is such that $p_{0}+\pi_{\ell}>0$;

(v) $\mu$ and $\eta$ are equal to two constants $\mu_{0}>0$ and $\eta_{0}$ satisfying $2 \mu_{0}+3 \eta_{0}>0$.

Notice the previous assumptions ensure that $\beta_{\ell}>0$.

Finally, the initial/boundary states involved in (4-7) are constrained by:

(vi) $D_{e}(t, x)>0$ for all $t \geq 0$ and $x \in\left[0, L_{x}\right]$;

(vii) $h_{e}(t, x)>q_{\ell}$ for all $t \geq 0$ and $x \in\left[0, L_{x}\right]$;

(viii) $h_{0}$ is such that $h_{0}(x, y=0)=h_{e}(t=0, x)$ for all $x \in\left[0, L_{x}\right]$ and $h_{0}(\boldsymbol{x})>q_{\ell}$ for all $\boldsymbol{x} \in\left[0, L_{x}\right] \times\left[0, L_{y}\right]$;

(ix) $\mathbf{u}_{0}$ is such that

$$
\left\{\begin{array}{l}
\nabla \cdot \mathbf{u}_{0}=\frac{\beta_{\ell}\left(p_{0}\right)}{p_{0}} \Phi(0, \boldsymbol{x}), \\
\mathbf{u}_{0}(x, y=0)=\mathbf{u}_{e}(t=0, x)
\end{array}\right.
$$

and satisfies $\mathrm{BC}(5)$ and (6).

Assumption (vi) corresponds to a nuclear power plant of PWR or BWR type: the flow is upward ${ }^{1}$. Assumption (vii) means that EOS $(2)$ is such that $\rho\left(h_{e}, p_{0}\right)$ is well-defined and positive. This enables to compute the inflow velocity $\mathbf{u}_{e}$ by

$$
u_{e}(t, x) \stackrel{\text { def }}{=} 0, \quad v_{e}(t, x)=\frac{D_{e}(t, x)}{\rho\left(h_{e}(t, x), p_{0}\right)} .
$$

Likewise, Hyp. (viii) leads to the existence of $\rho\left(h_{0}, p_{0}\right)$ through (2). Finally, Hyp. (ix) corresponds to the fact that the steady equation (1a) is initially satisfied, which means that initial conditions are well-prepared (see [3] for instance).

\footnotetext{
${ }^{1}$ The flow could be downward when the nuclear reactor is a material testing reactor. The present model still applies but with adapted BC.
} 


\section{WEAK FORMULATION AND NUMERICAL SCHEME}

To provide a numerical approximation of the solutions to System (1), we carry out a standard Finite Element approach in order to use the FrEeFEM ++ software [10]. As for the convective part, it can be treated by incorporating a trilinear form into the weak formulation as in [9]. However, in keeping with previous works $[2,3]$ and as FreEFEM ++ incorporates this feature, we decided to apply the method of characteristics [14].

Let us define the characteristic flow $\mathcal{X}$ as the solution of the ordinary differential equation (ODE)

$$
\left\{\begin{array}{l}
\frac{\mathrm{d} \mathcal{X}}{\mathrm{d} \tau}(\tau ; t, \boldsymbol{x})=\mathbf{u}(\tau, \mathcal{X}(\tau ; t, \boldsymbol{x})) \\
\mathcal{X}(t ; t, \boldsymbol{x})=\boldsymbol{x}
\end{array}\right.
$$

parametrized by $t \in \mathbb{R}$ and $\boldsymbol{x} \in \Omega$. A straightforward remark is that for any field $\zeta: \mathbb{R} \times \Omega \rightarrow \mathbb{R}^{p}$

$$
\left(\frac{\mathrm{d}}{\mathrm{d} \tau}[\zeta(\tau, \mathcal{X}(\tau ; t, \boldsymbol{x}))]\right)_{\mid \tau=t}=\partial_{t} \zeta(t, \boldsymbol{x})+(\mathbf{u}(t, \boldsymbol{x}) \cdot \nabla) \zeta(t, \boldsymbol{x})
$$

Hence, the convective part in Equations (1b) and (1c) can be approximated for any $\Delta t>0$ by

$$
\left[\partial_{t}+(\mathbf{u}(t, \boldsymbol{x}) \cdot \nabla)\right]\left(\begin{array}{l}
h \\
\mathbf{u}
\end{array}\right)(t, \boldsymbol{x}) \approx \frac{1}{\Delta t}\left[\left(\begin{array}{l}
h \\
\mathbf{u}
\end{array}\right)(t, \boldsymbol{x})-\left(\begin{array}{l}
h \\
\mathbf{u}
\end{array}\right)(t-\Delta t, \mathcal{X}(t-\Delta t ; t, \boldsymbol{x}))\right] .
$$

The algorithm induced by this technique thus consists in finding $(h, \mathbf{u}, \bar{p})(t, \cdot) \in\left(h_{e}+\mathcal{H}\right) \times\left(\mathbf{u}_{e}+\mathcal{U}\right) \times \mathrm{L}^{2}(\Omega)$ such that for all $(\theta, \mathbf{v}, \psi) \in \mathcal{H} \times \mathcal{U} \times \mathrm{L}^{2}(\Omega)$

$$
\begin{aligned}
& -\int_{\Omega} \psi \nabla \cdot \mathbf{u} \mathrm{d} \boldsymbol{x}=\frac{\beta_{\ell}}{p_{0}} \int_{\Omega} \Phi \psi \mathrm{d} \boldsymbol{x}, \\
& \text { - } \int_{\Omega} \frac{h(t, \boldsymbol{x})-h(t-\Delta t, \mathcal{X}(t-\Delta t ; t, \boldsymbol{x}))}{\Delta t} \theta(\boldsymbol{x}) \mathrm{d} \boldsymbol{x}=\int_{\Omega} \frac{\Phi(t, \boldsymbol{x})}{\rho\left(h(t, \boldsymbol{x}), p_{0}\right)} \theta(\boldsymbol{x}) \mathrm{d} \boldsymbol{x}, \\
& \text { - } \int_{\Omega} \rho\left(h(t, \boldsymbol{x}), p_{0}\right) \frac{\mathbf{u}(t, \boldsymbol{x})-\mathbf{u}(t-\Delta t, \mathcal{X}(t-\Delta t ; t, \boldsymbol{x}))}{\Delta t} \cdot \mathbf{v}(\boldsymbol{x}) \mathrm{d} \boldsymbol{x} \\
& +\frac{\mu_{0}}{2} \int_{\Omega}\left(\nabla \mathbf{u}+(\nabla \mathbf{u})^{T}\right)::\left(\nabla \mathbf{v}+(\nabla \mathbf{v})^{T}\right) \mathrm{d} \boldsymbol{x}+\eta_{0} \int_{\Omega}(\nabla \cdot \mathbf{u})(\nabla \cdot \mathbf{v}) \mathrm{d} \boldsymbol{x}-\int_{\Omega} \bar{p} \nabla \cdot \mathbf{v} \mathrm{d} \boldsymbol{x} \\
& =\int_{\Omega} \rho\left(h(t, \boldsymbol{x}), p_{0}\right) \mathbf{g} \cdot \mathbf{v}(\boldsymbol{x}) \mathrm{d} \boldsymbol{x},
\end{aligned}
$$

where

$$
\begin{aligned}
& \mathcal{H}=\left\{\theta \in \mathrm{H}^{1}(\Omega): \theta(x, 0)=0\right\} \\
& \mathcal{U}=\left\{\mathbf{v} \in\left(\mathrm{H}^{1}(\Omega)\right)^{2}: \mathbf{v}(x, 0)=\mathbf{0}, \mathbf{v} \cdot \mathbf{n}(0, y)=\mathbf{v} \cdot \mathbf{n}\left(L_{x}, y\right)=0\right\}
\end{aligned}
$$

Boundary integrals vanished either due to $\mathrm{BC}(5)$ and $(6 \mathrm{~b})$ or to the functional spaces including homogeneous versions of (4) and (6a).

The theoretical investigation of the weak formulation is not the topic of this paper. However, we just specify the choice of $\mathcal{H}$. The first reason is to ensure the existence of the trace of $h \in \mathcal{H}$ on $\{y=0\}$ in order to satisfy (4). The second one is that it enables to prove that $h-q_{\ell}>\min \left\{h_{0}-q_{\ell}\right\}$ through the weak formulation of the 


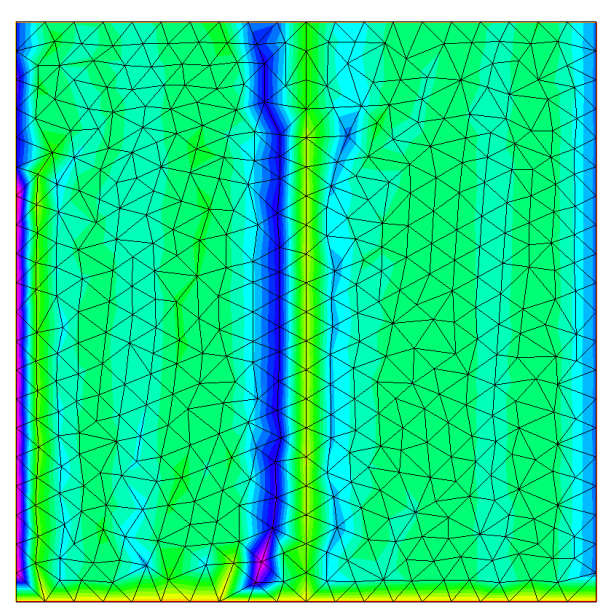

(a) Normalized error upon the density Scale ranges from $1.57 \times 10^{-6}$ (orange) to $5.83 \times 10^{-5}(\mathrm{red})$

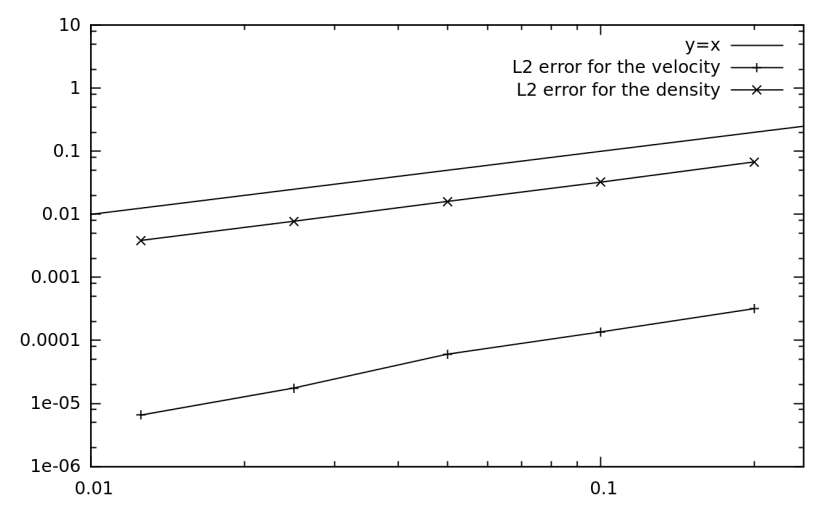

(b) Convergence curves: $\mathrm{L}^{2}$ errors with respect to the mesh size

FiguRE 1. Comparisons with exact solutions. Colors range from orange to yellow, to green, to blue and then to red.

transport equation and thus that $\rho\left(h, p_{0}\right)$ is positive and belongs to $\mathrm{L}^{\infty}(\Omega)$. The latter point shows that the integral $\int_{\Omega} \rho\left(h, p_{0}\right) \mathbf{u} \cdot \mathbf{v} \mathrm{d} \boldsymbol{x}$ is well-defined. We recall that $\rho\left(h, p_{0}\right)$ is defined by EOS (2).

As $\mathbf{u}$ is an unknown of the overall problem, the exact solution of ODE (8) cannot be achieved. There exist several numerical techniques to yield an approximation of $\mathcal{X}(t-\Delta t ; t, \boldsymbol{x})$ (see $[13,14]$ ). In the sequel, this approximation will be denoted by $\boldsymbol{\xi}$ no matter what method is used to compute it. The corresponding routine in FreEFEM++ is convect.

Given a time sampling $t^{0}, t^{1}, \ldots, t^{n}$, we consider the following semi-implicit discretization: find $\left(h^{n+1}, \mathbf{u}^{n+1}, \bar{p}^{n+1}\right) \in$ $\left(h_{e}+\mathcal{H}\right) \times\left(\mathbf{u}_{e}+\mathcal{U}\right) \times \mathrm{L}^{2}(\Omega)$ such that for all $(\theta, \mathbf{v}, \psi) \in \mathcal{H} \times \mathcal{U} \times \mathrm{L}^{2}(\Omega)$

$$
\begin{gathered}
\bullet \int_{\Omega} \nabla \cdot \mathbf{u}^{n+1} \psi \mathrm{d} \boldsymbol{x}=\frac{\beta_{\ell}}{p_{0}} \int_{\Omega} \Phi\left(t^{n}, \boldsymbol{x}\right) \psi \mathrm{d} \boldsymbol{x}, \quad \cdot \int_{\Omega} \frac{h^{n+1}-h^{n}\left(\boldsymbol{\xi}^{n}\right)}{\Delta t} \theta \mathrm{d} \boldsymbol{x}=\int_{\Omega} \frac{\Phi\left(t^{n}, \boldsymbol{x}\right)}{\rho\left(h^{n}, p_{0}\right)} \theta \mathrm{d} \boldsymbol{x}, \\
\bullet \int_{\Omega} \rho\left(h^{n}, p_{0}\right) \frac{\mathbf{u}^{n+1}-\mathbf{u}^{n}\left(\boldsymbol{\xi}^{n}\right)}{\Delta t} \cdot \mathbf{v} \mathrm{d} \boldsymbol{x}+\frac{\mu_{0}}{2} \int_{\Omega}\left(\nabla \mathbf{u}^{n+1}+\left(\nabla \mathbf{u}^{n+1}\right)^{T}\right)::\left(\nabla \mathbf{v}+(\nabla \mathbf{v})^{T}\right) \mathrm{d} \boldsymbol{x} \\
+\eta_{0} \int_{\Omega}\left(\nabla \cdot \mathbf{u}^{n+1}\right)(\nabla \cdot \mathbf{v}) \mathrm{d} \boldsymbol{x}-\int_{\Omega} \bar{p}^{n+1} \nabla \cdot \mathbf{v} \mathrm{d} \boldsymbol{x}=\int_{\Omega} \rho\left(h^{n}, p_{0}\right) \mathbf{g} \cdot \mathbf{v} \mathrm{d} \boldsymbol{x} .
\end{gathered}
$$

This weak formulation is discretized in space on a triangular mesh. It is then solved with the FE-software FreeFem ++ . We do not give more details about this step in the present paper. We only mention that the time step must be computed as a function of the mesh size in order to ensure convergence. ${ }^{2}$ 


\section{NumeriCAL EXAMPLES}

Numerical simulations of 1D single-phase flows are provided in [2]. Here, we focus on 2D flows and perform some simulations obtained by means of FREEFEM ++ (using $P_{1}$ Lagrange finite elements) applied to the discrete weak formulation presented in Section 3. These results are aimed at providing exploratory hints about the behaviour of solutions to our LMNC model in dimension 2.

Two data sets are considered: the first academic test enables to assess the numerical approach insofar as an analytic monodimensional solution must be recovered. The second test presents real $2 \mathrm{D}$ effects due to a nonvertical gravity field. Parameters are set as follows:

- Geometry of the domain: $L_{x}=L_{y}=1 \mathrm{~m}$.

- Parameters involved in EOS (2) for the pure liquid: $\gamma_{\ell}=2.35, \pi_{\ell}=10^{9} \mathrm{~Pa}, q_{\ell}=-1167.056 \times 10^{3} \mathrm{~J} \cdot \mathrm{kg}$.

- Reference value for pressure, gravity intensity and power density: $p_{0}=155 \times 10^{5} \mathrm{~Pa}, g=9.81 \mathrm{~m} \cdot \mathrm{s}^{-2}$, $\Phi_{0}=170 \times 10^{6} \mathrm{~W} \cdot \mathrm{m}^{-3}$.

- Inflow data: $h_{e}=1.236508 \times 10^{6} \mathrm{~J} \cdot \mathrm{K}^{-1}, v_{e}=5 \mathrm{~m} \cdot \mathrm{s}^{-1}$.

\subsection{Recovering $1 \mathrm{D}$ solutions}

In this first case, the setting is such that the analytic 1D solution derived in [3, Sect. 3.2] is also the solution of the $2 \mathrm{D}$ problem. It corresponds to the following choice for the parameters:

- Power density: $\Phi(t, \boldsymbol{x})=\Phi_{0}$.

- Gravity field: $\mathbf{g}=g \times(0,-1)$.

The result displayed on Figure 1(a) shows the pointwise error over the domain between the analytic solution $\rho\left(h^{\infty}(y), p_{0}\right)$ from [3, Remark 3.1] duplicated for each $x \in\left[0, L_{x}\right]$ and the numerical solution when the asymptotic state is reached (here $t=0.3 \mathrm{~s}$ ). The corresponding mesh is made of 952 triangles and 517 nodes while the mean error is about $10^{-6}$. The error is not uniform due to the unstructured mesh. We also performed a refinement process which leads to an order 1 convergence towards the monodimensional analytic solution - see Fig. 1(b).

The numerical strategy using FreEFem ++ and detailed above thus enables to recover the expected solution which legitimates the approach.

\subsection{Genuine 2D flows}

We now focus on a test which displays 2D phenomena. To do so, we modify the data:

- Power density: $\Phi(t, \boldsymbol{x})=\Phi_{0} \times 10^{4} \times \exp \left(\frac{-1}{r_{0}^{2}-\left|\boldsymbol{x}-\boldsymbol{x}_{0}\right|^{2}}\right) \mathbf{1}_{\left\{\left|x-x_{0}\right|<r_{0}\right\}}, \boldsymbol{x}_{0}=\left(\frac{1}{2}, \frac{1}{2}\right)$ and $r_{0}=0.4-$ see Fig. 2(a).

- Gravity field: $\mathbf{g}=g \frac{\sqrt{2}}{2} \times(1,-1)$.

This corresponds to a localized heating at the center of the core. We notice on Figs. 2(c) and (d) the effects of the convection compared to Figs. 3(a) and (b). The rise of temperature is not restricted to the support of $\Phi$ and a steady state can be reached. The influence of the nonvertical gravity field is also noticeable on Figs. 2(b) and (g). Indeed, we observe on Fig. 2(b) that the pressure increases at the low right part of the core which induces a dissymmetry on Fig. $2(\mathrm{~g})$. Fig. 2(f) also shows the $x$-component of the velocity is no longer

\footnotetext{
${ }^{2}$ The time step is set as follows: $\Delta t=\mathcal{C} \frac{\delta}{\left|u_{e}\right|}$ where $\delta$ is a characteristic mesh size and $\mathcal{C} \sim 1$ is a constant.
} 


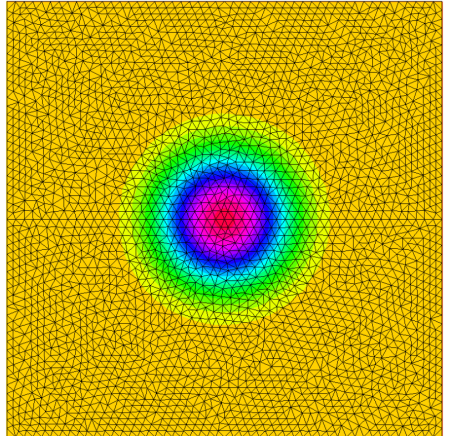

(a) Mesh and density power Scale ranges from $0 \mathrm{~W} \cdot \mathrm{m}^{-3} \quad$ (orange) to $3.38 \times 10^{9} \mathrm{~W} \cdot \mathrm{m}^{-3}$ (red).

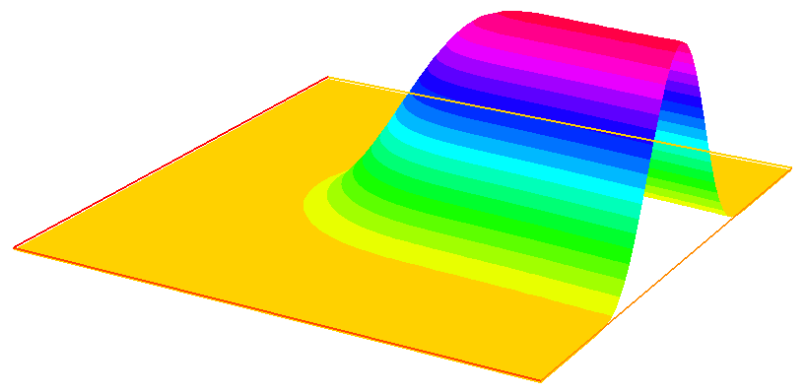

(c) Temperature - Scale ranges from $561 \mathrm{~K}$ (orange) to $621 \mathrm{~K}$ (red).

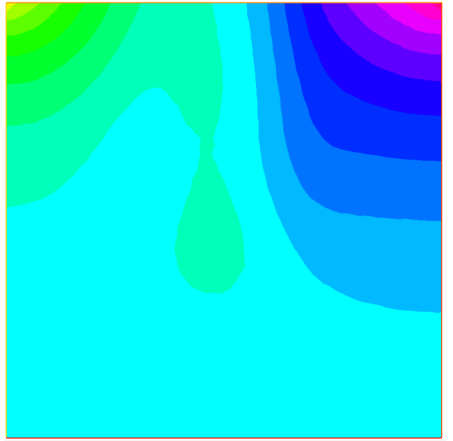

(e) Mach number - Scale ranges from $2.33 \times 10^{-3}$ (orange) to $3.3 \times 10^{-3}$ (red).

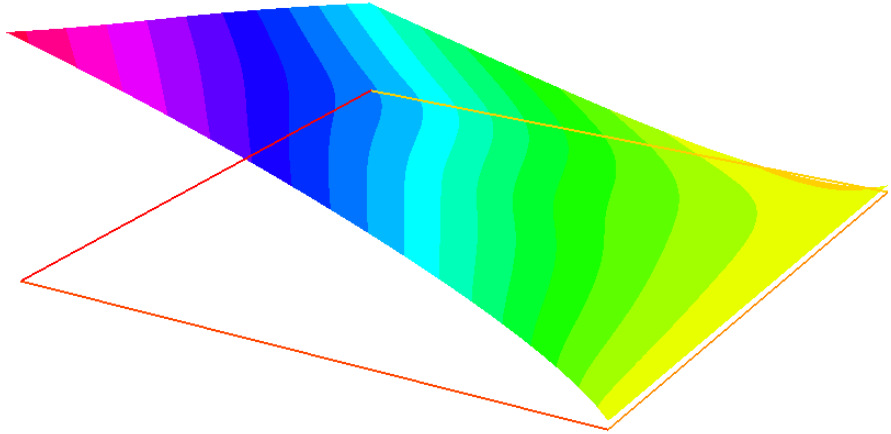

(b) $\bar{p}-\min _{\Omega} \bar{p}-$ Scale ranges from $0 \mathrm{~Pa}$ (orange) to $8459 \mathrm{~Pa}$ (red).

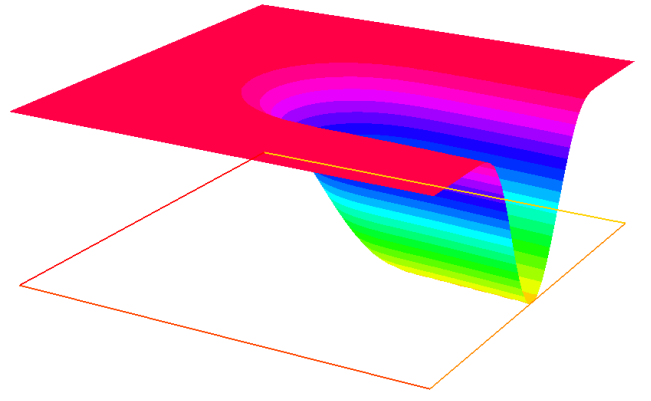

(d) Density - Scale ranges from $667 \mathrm{~kg} \cdot \mathrm{m}^{-3}$ (orange) to $738 \mathrm{~kg} \cdot \mathrm{m}^{-3}$ (red).

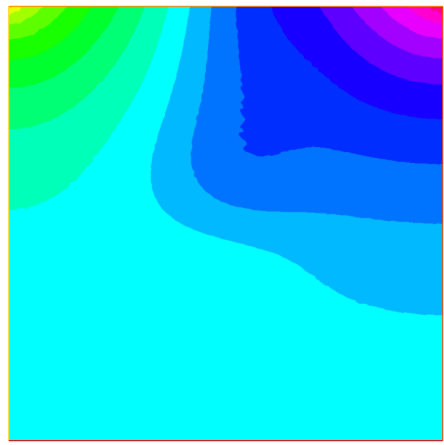

(g) Velocity: $y$-component - Scale ranges from $4.25 \mathrm{~m} \cdot \mathrm{s}^{-1} \quad$ (orange) to $5.92 \mathrm{~m} \cdot \mathrm{s}^{-1}$ (red)

Figure 2. Simulations of a confined heating. Colors range from orange to yellow, to green, to blue and then to red.

negligible. The fact still remains this experiment matches the low Mach number assumption as it is highlighted on Fig. 2(e). 


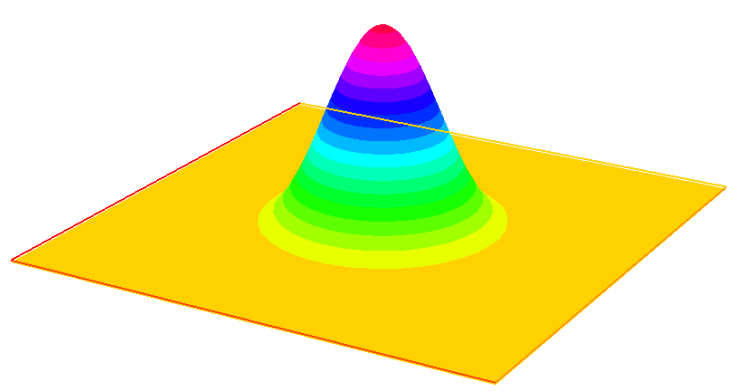

(a) Temperature - Scale ranges from $556 \mathrm{~K}$ (orange) to $806 \mathrm{~K}$ (red).

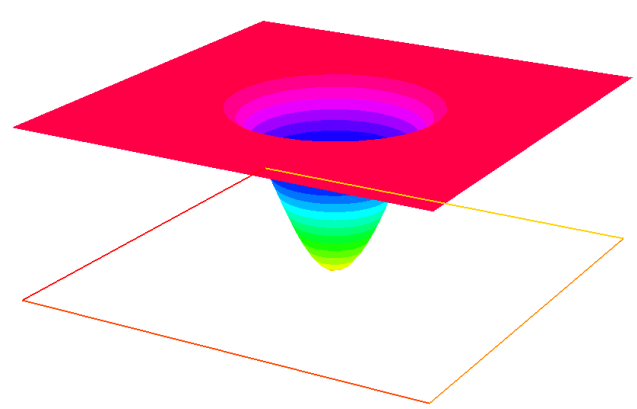

(b) Density - Scale ranges from $512 \mathrm{~kg} \cdot \mathrm{m}^{-3}$ (orange) to $742 \mathrm{~kg} \cdot \mathrm{m}^{-3}$ (red).

Figure 3. Test case: model without convective terms. Colors range from orange to yellow, to green, to blue and then to red.

\section{Conclusion \& Perspectives}

The 2D numerical results presented in this paper show that the Finite Element method coupled to a method of characteristics is suitable to solve a low Mach system which models the heat transfer in a nuclear core. It is achieved although the variety of nature of the equations involved in the system. The specific boundary conditions are correctly taken into account through the weak formulation.

This work is a first step in simulating the LMNC model after some 1D devoted papers $[2,3,7]$. Other steps must follow on, including the proof of numerical properties such as positivity preservation, convergence results and error estimates. Moreover, the modelling of the nuclear core will have to be improved, in particular by introducing phase transition using tabulated equations of state, which will be achieved in [8].

\section{REFERENCES}

[1] TRACE V5.0 Theory Manual, Field Equations, Solution Methods and Physical Models. Technical report, U.S. Nuclear Regulatory Commission, 2008.

[2] M. Bernard, S. Dellacherie, G. Faccanoni, B. Grec, O. Lafitte, T.-T. Nguyen, and Y. Penel. Study of low Mach nuclear core model for single-phase flow. ESAIM Proc., 38:118-134, 2012.

[3] M. Bernard, S. Dellacherie, G. Faccanoni, B. Grec, and Y. Penel. Study of low Mach nuclear core model for two-phase flows with phase transition I: stiffened gas law. Math. Model. Numer. Anal., 48(6):1639-1679, 2014.

[4] D. Bestion. The physical closure laws in the CATHARE code. Nucl. Eng. Des., 124(3):229-245, 1990.

[5] M. Braack and R. Rannacher. Adaptive finite element methods for low Mach number flows with chemical reactions. IWR, 1999.

[6] J.M. Delhaye. Thermohydraulique des réacteurs. EDP sciences, 2008.

[7] S. Dellacherie. On a low Mach nuclear core model. ESAIM Proc., 35:79-106, 2012.

[8] S. Dellacherie, G. Faccanoni, B. Grec, and Y. Penel. Study of low Mach nuclear core model for two-phase flows with phase transition II: tabulated EOS. In preparation.

[9] T. Fortin. Une méthode d'éléments finis à décomposition $L^{2}$ d'ordre élevé motivée par la simulation des écoulements diphasiques bas Mach. PhD thesis, Univ. Paris 6, 2006.

[10] F. Hecht. FreeFem,++ 3.25 edition, 2013.

[11] Olivier Hurisse. Couplage interfacial instationnaire de modèles diphasiques. PhD thesis, Univ. Aix-Marseille I, 2006.

[12] A. Majda and J. Sethian. The derivation and numerical solution of the equations for zero Mach number combustion. Combust. Sci. Technol., 42(3-4):185-205, 1985.

[13] Y. Penel. An explicit stable numerical scheme for the 1D transport equation. Discrete Contin. Dyn. Syst. Ser. S, 5(3):641-656, 2012. 
[14] O. Pironneau. On the transport-diffusion algorithm and its applications to the navier-stokes equations. Numer. Math., 38:309$332,1982$.

[15] G.I. Sivashinsky. Hydrodynamic theory of flame propagation in an enclosed volume. Acta Astronaut., 6:631-645, 1979.

[16] C. Taylor and P. Hood. Numerical solution of the Navier-Stokes equations using the finite element technique. Comput. ES Fluids, 1(1):73-100, 1973. 